\title{
Genetic Variants in the SORL1 Gene Are Associated with Age at Onset of Alzheimer Disease: A Survival Analysis
}

\author{
Ke-Sheng Wang, ${ }^{1}$ Xuefeng Liu, ${ }^{1}$ Liang Wang, ${ }^{1}$ David Briones, ${ }^{2}$ and Chun $\mathrm{Xu}^{2}$ \\ ${ }^{1}$ Department of Biostatistics and Epidemiology, College of Public Health, East Tennessee State University, P.O. Box 70259, \\ Lamb Hall, Johnson City, TN 37614-1700, USA \\ ${ }^{2}$ Departments of Psychiatry and Neurology and the Center of Excellence in Neuroscience, Paul L. Foster School of Medicine, \\ Texas Tech University Health Sciences Center, El Paso, TX 79905, USA
}

Correspondence should be addressed to Ke-Sheng Wang; wangk@etsu.edu

Received 17 January 2014; Revised 27 July 2014; Accepted 31 July 2014; Published 12 August 2014

Academic Editor: Jezabel V. López

Copyright (C) 2014 Ke-Sheng Wang et al. This is an open access article distributed under the Creative Commons Attribution License, which permits unrestricted use, distribution, and reproduction in any medium, provided the original work is properly cited.

Few studies focused on the association of SORL1 with the age at onset (AAO) of Alzheimer disease (AD). This study investigated the association of 43 SNPs in SORL1 with the AAO of AD by using the Kaplan-Meier survival analysis and the Cox proportional hazards model in SAS version 9.2 and linear regression model in PLINK software (791 AD patients and 782 controls). Both logrank test and Cox regression model showed that five SNPs (rs1784934, rs676759, rs560573, rs593769, and rs11218313) were associated with the AAO of AD in the male sample, while one SNP (rs17125558) was associated with the AAO of AD in the female sample $(P<0.05)$. SNP rs560573, previously associated with the risk of late-onset AD, showed the most association with the AAO in the male sample ( $P=0.0077$ for logrank test and $P=0.0117$ in the Cox model). The mean AAO was approximately 2.5 years earlier in individuals who were homozygous for the minor allele compared with those who had at least one major allele. Linear regression model showed that rs2282649 and rs726601 were associated with AAO in the whole sample $(P=0.0374$ and 0.0367 , resp.). These findings provide evidence of several genetic variants in SORL1 influencing the AAO of AD.

\section{Introduction}

Alzheimer disease (AD) is the most common form of dementia. Most often, AD is diagnosed in people over 65 years of age, although the less-prevalent early-onset Alzheimer's can occur much earlier [1]. In the United States, the prevalence of $\mathrm{AD}$ in 2000 was estimated to be about $1.6 \%$ in the $65-74$ age group, with the rate increasing to $19 \%$ in the $75-84$ age group and to $42 \%$ in the greater than 84 age group [2]. The World Health Organization estimated that, in 2005, 0.379\% of people worldwide had dementia, and the prevalence would increase to $0.441 \%$ in 2015 and to $0.556 \%$ in 2030 [3]. Another study estimated that, in $2006,0.40 \%$ of the world population (about 26.6 million) was afflicted by AD, which is predicted to affect 1 in 85 people globally by 2050 [4].

In addition to the disease risk, age at onset (AAO) of $\mathrm{AD}$ is also genetically influenced with an estimated heritability of about $42 \%[5,6]$. Genetic effects account for $57 \%-78 \%$ of the variance of AAO [7], while twin studies suggest that the heritability of $\mathrm{AD}$ exceeds $60 \%$ [8]. AD has a strong genetic predisposition (60-80\% of the attributable risk) [9].

The sortilin-related receptor (SORL1) gene is located at $11 \mathrm{q} 23.2-\mathrm{q} 24.2$ and is expressed as a $10.5 \mathrm{~kb}$ transcript in brain, spinal cord, and testis [10]. Rogaeva et al. [11] reported that inherited variants of the SORL1 neuronal sortilin-related receptor were associated with late-onset AD. Lee et al. [12] reported associations between various SNPs and haplotypes in the SORL1 gene and AD among a total of $296 \mathrm{AD}$ patients comprising 3 cohorts of African American, Caribbean Hispanic, and non-Hispanic white individuals. Their results suggested extensive allelic heterogeneity in SORL1, with specific SNPs associated with specific groups. Cellini et al. [13] concluded that their findings confirmed the association between SORL1 and late-onset AD. Reitz et al. [14] also reported that their findings provided confirmatory evidence of the association of multiple SORL1 variants with AD risk. However, few studies have focused on the association of SORL1 with the AAO of AD $[12,15]$. This study explored 
TABLE 1: Descriptive characteristics of cases and controls.

\begin{tabular}{lcccc}
\hline Variable & Total patients & Male patients & Female patients & Control \\
\hline Sample size $(n)$ & 791 & 334 & 457 & 782 \\
Mean AAO (years \pm SD) & $72.3( \pm 8.5)$ & $71.8( \pm 8.3)$ & 73 & NA \\
Median AAO (years) & 73 & $44-90$ & $73.6)$ & NA \\
Range of age at onset (years) & $40-97$ & $77.5( \pm 8.4)$ & NA & 707 \\
Mean age at entry (years \pm SD) & $77.6( \pm 8.6)$ & 79 & $78.2( \pm 8.7)$ & 79 \\
Median age at entry (years) & 79 & $49-94$ & $43-100$ & $79.4( \pm 7.9)$ \\
Range of age at entry (years) & $43-100$ & $48-94$ \\
\hline
\end{tabular}

the association of SORL1 with the AAO of AD in a Canadian sample by using the Kaplan-Meier survival analysis, the Cox proportional hazards model, and linear regression model.

\section{Materials and Methods}

2.1. Subjects. Seven hundred and ninety-one patients with $\mathrm{AD}$ and 782 controls with complete genotype and phenotype information in a Canadian sample were selected from the Multi-Site Collaborative Study for GenotypePhenotype Associations in Alzheimer's Disease and the Longitudinal Follow-Up of Genotype-Phenotype Associations in Alzheimer's Disease and the Neuroimaging Component of Genotype-Phenotype Associations in Alzheimer's Disease (dbGaP Study Accession: phs000219.v1.p1). The details about these subjects were described elsewhere $[16,17]$. Genotyping was conducted using the Affymetrix $500 \mathrm{~K}$ Set comprising the Mapping250 K_Nsp and Mapping250 K_Sty Arrays (total 262,264 SNPs). We investigated the genetic associations of 43 single-nucleotide polymorphisms (SNPs) within the SORL1 gene with the $\mathrm{AAO}$ of $\mathrm{AD}$.

2.2. Statistical Methods. Hardy-Weinberg equilibrium was tested for all SNPs using the controls by using Haploview software [18]. Then, minor allele frequency (MAF) was determined for each SNP. The assessment of the association between genotypes of each SNP and AAO was initially performed using the logrank test (a nonparametric test) in Kaplan-Meier (KM) survival analysis. The KM survival curves were used to plot the survival function. When a significant value was obtained in the KM testing, the Cox proportional hazards model (a semiparametric model) was used to confirm the findings of KM analysis. Hazard ratio (HR) and 95\% CI were calculated with Cox proportional hazards analysis of SNPs. Descriptive statistics for AAO and survival analyses were conducted with SAS statistical software, version 9.2 (SAS Institute, Cary, NC, USA).

To test for association with AAO as a quantitative trait, linear regression analysis was performed by PLINK 1.07 [19] to obtain the regression coefficient $(\beta)$ and Wald test asymptotic $P$ value. For statistical significance, empirical $P$ values were generated by 100,000 permutation tests using Max (T) permutation procedure implemented in PLINK. This procedure allows calculation of the pointwise estimates of an individual SNP's significance (empirical pointwise $P$ values). Pairwise linkage disequilibrium (LD) statistics $\left(D^{\prime}\right)$ were assessed for the controls using Haploview [18]. We identified haplotype blocks based on solid spine of LD using Haploview [18]. Then we chose several SNPs within blocks including the associated SNPs for further analyses. Haplotype analysis based on a slide-window was performed using PLINK. To perform gene-based analysis, we constructed SNP sets based on haplotpye blocks; then we perform set-based tests using PLINK.

\section{Results}

3.1. Subjects Characteristics. The demographic characteristics of the subjects in the study are detailed in Table 1 . The mean AAO for the cases was 72.3 years (71.8 years for the males and 72.6 years for the females, resp.). The mean age at entry into study was 77.6 years for cases ( 77.5 years for the males and 78.2 years for the females, resp.) and 73.4 years for the controls.

3.2. Survival Analyses. Of the 43 SNPs, none was associated with the AAO of AD in the whole sample using the logrank test. Interestingly, five SNPs were associated with the AAO of $\mathrm{AD}$ in the male sample, while one SNP was associated with the AAO of AD in the female sample $(P<0.05)$. The results of the top 6 associated SNPs based on the logrank test are summarized in Table 2. All 6 SNPs were in Hardy-Weinberg equilibrium in the controls. The most statistically significant associated SNPs were rs560573 $(P=0.0077)$ and rs593760 $(P=0.0077)$ in the male sample.

Cox proportional models further confirmed the associations of these 6 SNPs (Table 3). SNPs rs560573 and rs593769 showed the most association with AAO in the Cox model with $\mathrm{HR}=1.47(95 \% \mathrm{CI}=1.09-1.97)$. The mean AAO was approximately 2.5 years earlier for individuals (TT for rs560573 and CC for rs593769) who were homozygous for the minor allele compared with those (AA or AT for rs560573 and CT + TT for rs593769) who had at least one major allele. Figure 1 shows the survival function for different genotypes of SNP rs560573. The median of AAO was 71 years for individuals with TT genotype and 73 years for individuals with AA or AT genotypes.

3.3. Linear Regression Analysis. The results of linear regression model are summarized in Table 4. Single-marker analysis showed that rs2282649 and rs726601 were associated with AAO in the whole sample ( $P=0.0374$ and 0.0367 , resp.) and rs11218313 was associated with AAO in the male sample $(P=0.0318)$. For the $\operatorname{rs} 11218313(\beta=2.82)$, the mean AAO in male was approximately 2.8 years earlier for the minor allele $G$ 
TABLE 2: Top 6 SNPs associated with the age at onset of Alzheimer's disease using the logrank test.

\begin{tabular}{lccccccc}
\hline SNP & Position & $\mathrm{Al}^{\mathrm{a}}$ & $\mathrm{MAF}^{\mathrm{b}}$ & $\mathrm{HWE}^{\mathrm{c}}$ & Logrank-all $^{\mathrm{d}}$ & Logrank-male $^{\mathrm{e}}$ & Logrank-female $^{\mathrm{f}}$ \\
\hline rs1784934 & 121467284 & $\mathrm{C}$ & 0.36 & 0.35 & 0.288 & 0.040 & 0.96 \\
rs676759 & 121488556 & $\mathrm{G}$ & 0.40 & 0.49 & 0.154 & 0.008 & 0.933 \\
rs560573 & 121490175 & $\mathrm{~T}$ & 0.39 & 0.59 & 0.176 & 0.0077 & 0.836 \\
rs593769 & 121493294 & $\mathrm{C}$ & 0.39 & 0.84 & 0.152 & 0.0077 & 0.933 \\
rs11218313 & 121512162 & $\mathrm{G}$ & 0.09 & 0.38 & 0.185 & 0.0317 & 0.89 \\
rs17125558 & 121003094 & $\mathrm{~T}$ & 0.03 & 0.71 & 0.0715 & 0.758 & 0.039 \\
\hline
\end{tabular}

${ }^{\mathrm{a}}$ Minor allele; ${ }^{\mathrm{b}}$ minor allele frequency; ${ }^{\mathrm{c}}$ Hardy-Weinberg equilibrium $P$ value; ${ }^{\mathrm{d}} P$ value was based on logrank test for the whole sample; ${ }^{\mathrm{e}} P$ value was based on the logrank test for the males; ${ }^{\mathrm{f}} P$ value was based on the logrank test for the females.

TABLE 3: Top 6 SNPs associated with the age at onset of Alzheimer's disease using the Cox proportional hazards model.

\begin{tabular}{|c|c|c|c|c|c|c|c|c|c|c|}
\hline SNP & Male & Mean & $\mathrm{HR}^{\mathrm{a}}$ & $95 \% \mathrm{CI}^{\mathrm{b}}$ & $P$ & Female & Mean & $\mathrm{HR}$ & $95 \% \mathrm{CI}$ & $P$ \\
\hline \multicolumn{11}{|l|}{ rs1784934 } \\
\hline $\mathrm{AA}$ & 42 & 70.6 & 1.39 & \multirow{2}{*}{$1.00-1.93$} & \multirow{2}{*}{0.049} & 59 & 72.9 & 1.01 & \multirow{2}{*}{$0.76-1.32$} & \multirow{2}{*}{0.962} \\
\hline $\mathrm{AG}+\mathrm{GG}$ & 292 & 72.0 & 1.00 & & & 398 & 73.5 & 1.00 & & \\
\hline \multicolumn{11}{|l|}{ rs676759 } \\
\hline GG & 53 & 69.8 & 1.46 & \multirow{2}{*}{$1.09-1.97$} & \multirow{2}{*}{0.0121} & 65 & 73.4 & 0.99 & \multirow{2}{*}{$0.76-1.29$} & \multirow{2}{*}{0.937} \\
\hline $\mathrm{AA}+\mathrm{AG}$ & 281 & 72.2 & & & & 392 & 72.5 & 1.00 & & \\
\hline \multicolumn{11}{|l|}{ rs560573 } \\
\hline $\mathrm{TT}$ & 53 & 69.7 & 1.47 & \multirow{2}{*}{$1.09-1.97$} & \multirow{2}{*}{0.0117} & 63 & 73.5 & 0.97 & \multirow{2}{*}{$0.74-1.27$} & \multirow{2}{*}{0.845} \\
\hline $\mathrm{AA}+\mathrm{AT}$ & 281 & 72.2 & 1.00 & & & 394 & 72.4 & 1.00 & & \\
\hline \multicolumn{11}{|l|}{ rs593769 } \\
\hline $\mathrm{CC}$ & 53 & 69.7 & 1.47 & \multirow{2}{*}{$1.09-1.97$} & \multirow{2}{*}{0.0117} & 65 & 73.4 & 0.99 & \multirow{2}{*}{$0.76-1.29$} & \multirow{2}{*}{0.937} \\
\hline $\mathrm{CT}+\mathrm{TT}$ & 281 & 72.2 & 1.00 & & & 392 & 72.5 & 1.00 & & \\
\hline \multicolumn{11}{|l|}{ rs11218313 } \\
\hline $\mathrm{AG}+\mathrm{GG}$ & 45 & 74.2 & 0.72 & \multirow{2}{*}{$0.53-0.99$} & \multirow{2}{*}{0.0459} & 80 & 72.6 & 0.98 & \multirow{2}{*}{$0.77-1.26$} & \multirow{2}{*}{0.897} \\
\hline AA & 289 & 71.4 & 1.00 & & & 377 & 72.6 & 1.00 & & \\
\hline \multicolumn{11}{|l|}{ rs17125558 } \\
\hline $\mathrm{CT}+\mathrm{TT}$ & 14 & 71.4 & 1.08 & \multirow{2}{*}{$0.63-1.85$} & \multirow{2}{*}{0.772} & 25 & 70.1 & 1.50 & \multirow{2}{*}{$1.00-2.25$} & \multirow{2}{*}{0.0494} \\
\hline $\mathrm{CC}$ & 320 & 71.8 & 1.00 & & & 432 & 72.7 & 1.00 & & \\
\hline
\end{tabular}

${ }^{\mathrm{a}} \mathrm{HR}$ refers to hazard ratio; ${ }^{\mathrm{b}} \mathrm{CI}$ refers to confidence interval.

TABLE 4: Association results for 8 SNPs and the age at onset of Alzheimer's disease using the linear regression model.

\begin{tabular}{lccccccccccc}
\hline SNP & Position & $\mathrm{Al}^{\mathrm{a}}$ & $\beta$-all $^{\mathrm{b}}$ & $P$-all & EMP1 $^{\mathrm{d}}$ & $\beta$-male $^{\mathrm{e}}$ & $P$-male & EMP-male $^{\mathrm{g}}$ & $\beta$-female & $P$-female & EMP-female $^{\mathrm{j}}$ \\
\hline rs1784934 & 121467284 & $\mathrm{C}$ & 0.08 & 0.865 & 0.865 & -0.71 & 0.296 & 0.295 & 0.65 & 0.261 \\
rs676759 & 121488556 & $\mathrm{G}$ & 0.14 & 0.749 & 0.752 & -0.78 & 0.240 & 0.242 & 0.85 & 0.144 \\
rs560573 & 121490175 & $\mathrm{~T}$ & 0.13 & 0.764 & 0.767 & -0.77 & 0.238 & 0.24 & 0.85 & 0.148 \\
rs593769 & 121493294 & $\mathrm{C}$ & 0.12 & 0.784 & 0.788 & -0.79 & 0.223 & 0.225 & 0.83 & 0.152 & 0.147 \\
rs11218313 & 121512162 & $\mathrm{G}$ & 0.96 & 0.225 & 0.225 & 2.82 & 0.0326 & 0.0318 & -0.09 & 0.93 & 0.935 \\
rs2282649 & 121608249 & $\mathrm{~T}$ & 0.97 & 0.0394 & 0.0374 & 0.67 & 0.359 & 0.365 & 1.15 & 0.0636 & 0.0637 \\
rs726601 & 121610698 & $\mathrm{~T}$ & 0.96 & 0.0382 & 0.0367 & 0.77 & 0.287 & 0.296 & 1.06 & 0.083 & 0.0844 \\
rs17125558 & 121003094 & $\mathrm{~T}$ & -1.78 & 0.648 & 0.653 & -0.46 & 0.881 & 0.883 & -2.61 & 0.67 & 0.657 \\
\hline
\end{tabular}

${ }^{\mathrm{a}}$ Minor allele; ${ }^{\mathrm{b}} \beta$ refers to the regression coefficient of the linear regression for all patients; ${ }^{\mathrm{c}} P$ value refers to the Wald test asymptotic $P$ value for all patients; ${ }^{\mathrm{d}}$ empirical $P$ value for all patients generated by 100,000 permutation tests using Max (T) permutation procedure; ${ }^{\mathrm{e}} \beta$ refers to the regression coefficient of the linear regression for male patients; ${ }^{\mathrm{f}} P$ value refers to the Wald test asymptotic $P$ value for male patients; ${ }^{\mathrm{g}}$ empirical $P$ value for male patients generated by 100,000 permutation tests using $\operatorname{Max}(\mathrm{T})$ permutation procedure; ${ }^{\mathrm{h}} \beta$ refers to the regression coefficient of the linear regression for female patients; ${ }^{\mathrm{i}} P$ value refers to the Wald test asymptotic $P$ value for female patients; ${ }^{j}$ empirical $P$ value for female patients generated by 100,000 permutation tests using Max (T) permutation procedure. 
TABLE 5: Haplotype analysis for the whole sample.

\begin{tabular}{lcccc}
\hline \multicolumn{2}{c}{ Haplotype } & Frequency $^{\mathrm{a}}$ & $\beta^{\mathrm{b}}$ & $P$ value $^{\mathrm{c}}$ \\
\hline $\mathrm{rs} 2282648$ & rs2282649 & & & \\
$\mathrm{T}$ & $\mathrm{T}$ & 0.30 & 0.97 & 0.0394 \\
$\mathrm{~T}$ & $\mathrm{C}$ & 0.02 & -0.48 & 0.744 \\
$\mathrm{C}$ & $\mathrm{C}$ & 0.68 & -0.89 & 0.0554 \\
$\mathrm{rs} 2282649$ & rs726601 & & & \\
$\mathrm{T}$ & $\mathrm{T}$ & 0.29 & 1.03 & 0.0294 \\
$\mathrm{C}$ & $\mathrm{T}$ & 0.02 & -0.30 & 0.842 \\
$\mathrm{C}$ & $\mathrm{C}$ & 0.69 & -0.91 & 0.0502 \\
\hline
\end{tabular}

${ }^{a}$ Haplotype frequency in the sample.

${ }^{\mathrm{b}} \beta$ refers to the regression coefficient of the linear regression.

${ }^{\mathrm{c}} P$ value for the haplotype.

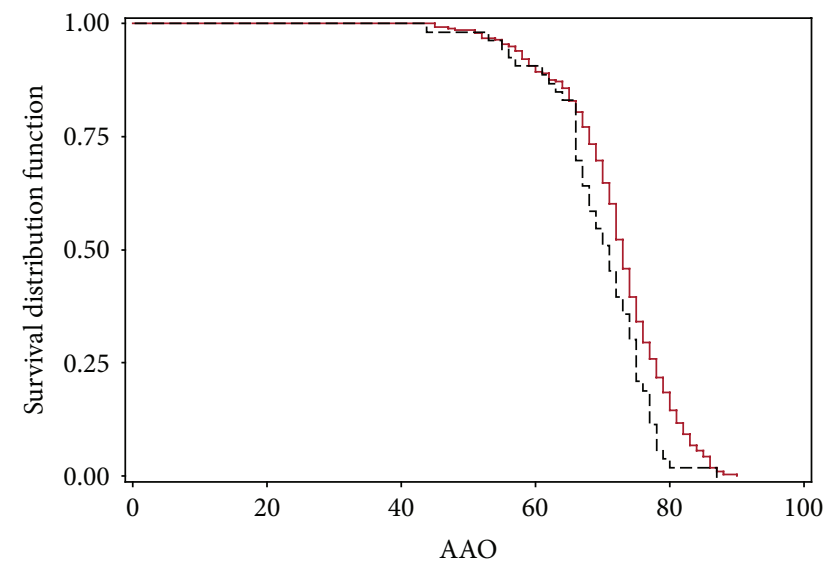

STRATA

- - rs560573 $=0$

- rs560573 = 1

FIGURE 1: Survival function by genotype of rs560573 $0=\mathrm{TT}$ Genotype, 1 = AA + AT Genotypes.

compared to major allele. All 3 SNPs had empirical pointwise $P$ values $<0.05$ using a permutation procedure (Table 4 ).

Using Haploview software, we identified 4 haplotype blocks for 43 SNPs. Haplotype analyses for the whole sample (Table 5) showed that the T-T haplotype from rs2282648 and rs2282649 $\left(D^{\prime}=1\right)$ was associated with AAO $(P=0.0394)$, and the T-T haplotype from rs2282649 and rs726601 $\left(D^{\prime}=\right.$ $0.98)$ was associated with AAO $(P=0.0294)$. Furthermore, haplotype analyses for the male sample (Table 6) showed that the C-G haplotype from rs668387 and $\operatorname{rs} 12218313\left(D^{\prime}=1\right)$ was associated with AAO $(P=0.0326)$, and the $\mathrm{C}-\mathrm{G}$ haplotype from rs1218313 and rs626885 $\left(D^{\prime}=0.91\right)$ was associated with $\mathrm{AAO}(P=0.0326)$.

Based on the 4 haplotype blocks, we built 4 set SNPs. We performed set-based tests using PLINK for the whole sample and also for the male sample. We did not find any significant associations for the set-based analyses (data not shown).
TABLE 6: Haplotype analysis for the male sample.

\begin{tabular}{lcccc}
\hline \multicolumn{2}{c}{ Haplotype } & Frequency $^{\mathrm{a}}$ & $\beta^{\mathrm{b}}$ & $P$ value $^{\mathrm{c}}$ \\
\hline rs668387 & rs11218313 & & & \\
$\mathrm{C}$ & $\mathrm{G}$ & 0.08 & 2.82 & 0.0326 \\
$\mathrm{~T}$ & $\mathrm{~A}$ & 0.45 & -0.28 & 0.66 \\
$\mathrm{C}$ & $\mathrm{A}$ & 0.47 & -0.35 & 0.51 \\
rs11218313 & rs626885 & & & \\
$\mathrm{A}$ & $\mathrm{C}$ & 0.43 & -0.09 & 0.878 \\
$\mathrm{C}$ & $\mathrm{G}$ & 0.07 & 2.82 & 0.0326 \\
$\mathrm{~A}$ & $\mathrm{G}$ & 0.5 & -0.56 & 0.38 \\
\hline $\mathrm{a}$ & & & &
\end{tabular}

${ }^{a}$ Haplotype frequency in the sample.

${ }^{\mathrm{b}} \beta$ refers to the regression coefficient of the linear regression.

${ }^{c} P$ value for the haplotype.

\section{Discussion}

In the present study we explored the association of 43 SNPs in the SORL1 gene with the AAO of AD by using the KaplanMeier survival analysis, the Cox proportional hazard model, and the linear regression model. Using the Kaplan-Meier survival analysis and Cox proportional hazard we found that five SNPs were significantly associated with the AAO of AD in the male sample and one SNP was associated with the AAO of $\mathrm{AD}$ in the female sample $(P<0.05)$. Linear regression model showed that two SNPs (rs2282649 and rs726601) were associated with AAO in the whole sample. SNP rs11218313 was associated with AAO in the male sample using logrank test and Cox model was also associated with AAO in the male sample in the linear model. Haplotype analyses in the linear model further supported the associations of single-marker analyses.

It has been reported that SORL1 gene is consistently downregulated approximately 2 -fold in lymphoblasts from patients with $\mathrm{AD}$, and the structure and function of SORL1 as a mosaic ApoE receptor strongly suggest that it plays a role in AD [20]. Sager et al. [21] also found a correlation between decreased SORL1 expression and impaired cognitive function among 34 individuals, including 10 patients with $\mathrm{AD}, 15$ with mild cognitive impairment, and 9 controls, suggesting that decreased SORL1 expression reflects cognitive performance and may predispose individuals with mild cognitive impairment to the development of AD.

Several previous studies have reported that SORL1 gene is associated with the risk of $\mathrm{AD}[11,12,14]$; however, few studies have focused on the association of SORL1 with the $\mathrm{AAO}$ of $\mathrm{AD}$. Lee et al. [12] examined the associations of 7 SNPs in SORL1 gene with the AAO of AD and found that 2 SNPs (rs536360 and rs556349) were associated with the $\mathrm{AAO}$ of $\mathrm{AD}$. Another study [15] investigated the association of 4 SORL1 genetic variants (rs2070045, SORL1-18ex26, rs3824968, and rs1010159) with the AAO of AD and found that SORL1-18ex26 was associated with an earlier AAO of $\mathrm{AD}$ in a German population. In the present study, of the 43 SNPs, none was associated with the AAO of $\mathrm{AD}$ in the whole sample using the logrank test; however, two SNPs were associated with AAO in the whole sample in the linear model. Furthermore, we found that five SNPs (rs1784934, rs676759, 
rs560573, rs593769, and rs11218313) were statistically significantly associated with the AAO of AD in the male sample, and one SNP (rs17125558) was associated with the AAO of AD in the female sample $(P<0.05)$ using survival analyses. Several studies examined rs560573 with the risk of $\mathrm{AD}[11,12,14,22$, 23 ] and one study found that rs 560573 was associated with the risk of $\mathrm{AD}$ [23]. Previous studies did not find the associations of other SNPs (rs676759, rs593769, rs11218313, and rs17125558) with the risk of $\mathrm{AD}[22]$.

There are a number of strengths in this study. First, our sample size was relatively large for this type of study. Second, we used the logrank test to examine the association with the AAO and further confirmed the results using the Cox proportional model. Furthermore, we conducted linear regression analyses. In addition, we detected gender differences. We also realized some limitations in this study. First, our current findings might be spurious or subject to type I error. Second, these findings need to be replicated in additional samples.

\section{Conclusions}

Our results demonstrate that genetic variants in SORL1 gene are associated with the AAO of AD. These findings may serve as a resource for replication in other populations. Future functional study of this gene may help to better characterize the genetic architecture of the AAO of AD.

\section{Conflict of Interests}

The authors declare that there is no conflict of interests regarding the publication of this paper.

\section{Acknowledgments}

We acknowledge the NIH GWAS Data Repository, the Contributing Investigator(s) who contributed the phenotype data and DNA samples from his/her original study, and the primary funding organization that supported the contributing study "Multi-Site Collaborative Study for GenotypePhenotype Associations in Alzheimer's Disease and Longitudinal Follow-Up of Genotype-Phenotype Associations in Alzheimer's Disease and Neuroimaging Component of Genotype-Phenotype Associations in Alzheimer's Disease." The genotypic and associated phenotypic data used in the study, "Multi-Site Collaborative Study for GenotypePhenotype Associations in Alzheimer's Disease (GenADA)" were provided by the GlaxoSmithKline, R\&D Limited. The datasets used for analyses described in this paper were obtained from dbGaP at http://www.ncbi.nlm.nih.gov/gap through dbGaP Accession no. phs000219.v1.p1.

\section{References}

[1] R. Brookmeyer, S. Gray, and C. Kawas, "Projections of Alzheimer's disease in the United States and the public health impact of delaying disease onset," American Journal of Public Health, vol. 88, no. 9, pp. 1337-1342, 1998.
[2] L. E. Hebert, P. A. Scherr, J. L. Bienias, D. A. Bennett, and D. A. Evans, "Alzheimer disease in the US population: prevalence estimates using the 2000 census," Archives of Neurology, vol. 60, no. 8, pp. 1119-1122, 2003.

[3] World Health Organization, Neurological Disorders: Public Health Challenges, World Health Organization, Geneva, Switzerland, 2006.

[4] R. Brookmeyer, E. Johnson, K. Ziegler-Graham, and M. H. Arrighi, "Forecasting the global burden of Alzheimer's disease," Alzheimer's and Dementia, vol. 3, no. 3, pp. 186-191, 2007.

[5] E. W. Daw, H. Payami, E. J. Nemens et al., "The number of trait loci in late-onset Alzheimer disease," The American Journal of Human Genetics, vol. 66, no. 1, pp. 196-204, 2000.

[6] Y. J. Li, W. K. Scott, D. J. Hedges et al., "AAO in two common neurodegenerative diseases is genetically controlled," The American Journal of Human Genetics, vol. 70, pp. 985-993, 2002.

[7] N. L. Pedersen, S. F. Posner, and M. Gatz, "Multiple-threshold models for genetic influences on age of onset for Alzheimer disease: findings in Swedish twins," American Journal of Medical Genetics: Neuropsychiatric Genetics, vol. 105, no. 8, pp. 724-728, 2001.

[8] J. W. Ashford and J. A. Mortimer, "Non-familial Alzheimer's disease is mainly due to genetic factors," Journal of Alzheimer's Disease, vol. 4, no. 3, pp. 169-177, 2002.

[9] M. Gatz, C. A. Reynolds, L. Fratiglioni et al., "Role of genes and environments for explaining Alzheimer disease," Archives of General Psychiatry, vol. 63, no. 2, pp. 168-174, 2006.

[10] L. Jacobsen, P. Madsen, S. K. Moestrup et al., "Molecular characterization of a novel human hybrid-type receptor that binds the alpha(2)-macroglobulin receptor-associated protein," The Journal of Biological Chemistry, vol. 271, no. 49, pp. 3137931383, 1996.

[11] E. Rogaeva, Y. Meng, J. H. Lee et al., “The neuronal sortilinrelated receptor SORL1 is genetically associated with Alzheimer disease," Nature Genetics, vol. 39, no. 2, pp. 168-177, 2007.

[12] J. H. Lee, R. Cheng, N. Schupf et al., "The association between genetic variants in SORL1 and Alzheimer disease in an urban, multiethnic, community-based cohort," Archives of Neurology, vol. 64 , no. 4 , pp. 501-506, 2007.

[13] E. Cellini, A. Tedde, S. Bagnoli et al., "Implication of sex and SORL1 variants in Italian patients with Alzheimer disease," Archives of Neurology, vol. 66, no. 10, pp. 1260-1266, 2009.

[14] C. Reitz, R. Cheng, E. Rogaeva et al., "Meta-analysis of the association between variants in SORL1 and Alzheimer disease," Archives of Neurology, vol. 68, no. 1, pp. 99-106, 2011.

[15] H. Kölsch, F. Jessen, J. Wiltfang et al., "Association of SORL1 gene variants with Alzheimer's disease," Brain Research, vol. 1264, pp. 1-6, 2009.

[16] H. Li, S. Wetten, L. Li et al., "Candidate single-nucleotide polymorphisms from a genomewide association study of Alzheimer disease," Archives of Neurology, vol. 65, no. 1, pp. 45-53, 2008.

[17] N. Filippini, A. Rao, S. Wetten et al., "Anatomically-distinct genetic associations of APOE eopen 4 allele load with regional cortical atrophy in Alzheimer's disease," NeuroImage, vol. 44, no. 3, pp. 724-728, 2009.

[18] J. C. Barrett, B. Fry, J. Maller, and M. J. Daly, "Haploview: analysis and visualization of LD and haplotype maps," Bioinformatics, vol. 21, no. 2, pp. 263-265, 2005.

[19] S. Purcell, B. Neale, K. Todd-Brown et al., "PLINK: a tool set for whole-genome association and population-based linkage analyses," The American Journal of Human Genetics, vol. 81, no. 3, pp. 559-575, 2007. 
[20] C. R. Scherzer, K. Offe, M. Gearing et al., "Loss of apolipoprotein E receptor LR11 in Alzheimer disease," Archives of Neurology, vol. 61, no. 8, pp. 1200-1205, 2004.

[21] K. L. Sager, J. Wuu, S. E. Leurgans et al., "Neuronal LR11/SorLA expression is reduced in mild cognitive impairment," Annals of Neurology, vol. 62, no. 6, pp. 640-647, 2007.

[22] Y. Meng, J. H. Lee, R. Cheng, P. St George-Hyslop, R. Mayeux, and L. A. Farrer, "Association between SORL1 and Alzheimer's disease in a genome-wide study," NeuroReport, vol. 18, no. 17, pp. 1761-1764, 2007.

[23] K. Bettens, N. Brouwers, S. Engelborghs, P. P. de Deyn, C. Van Broeckhoven, and K. Sleegers, "SORL1 is genetically associated with increased risk for late-onset Alzheimer disease in the Belgian population.," Human mutation, vol. 29, no. 5, pp. 769770, 2008. 


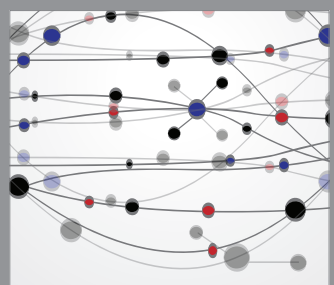

The Scientific World Journal
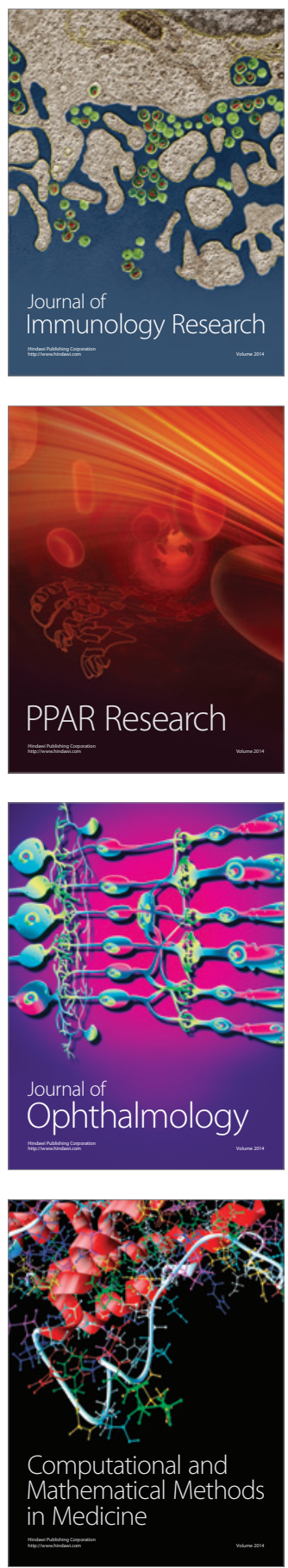

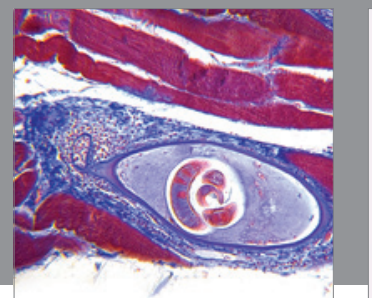

Gastroenterology

Research and Practice
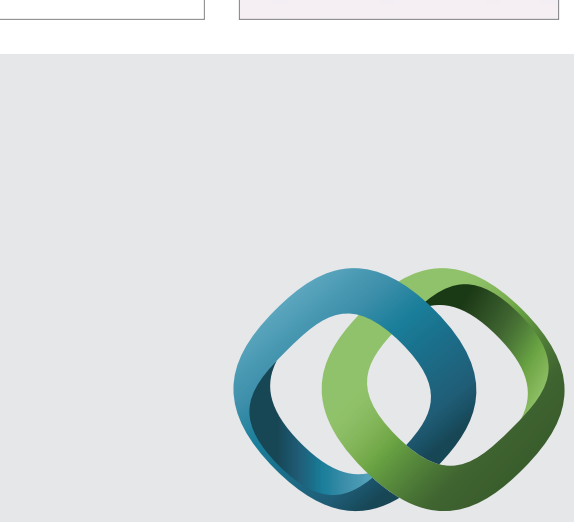

\section{Hindawi}

Submit your manuscripts at

http://www.hindawi.com
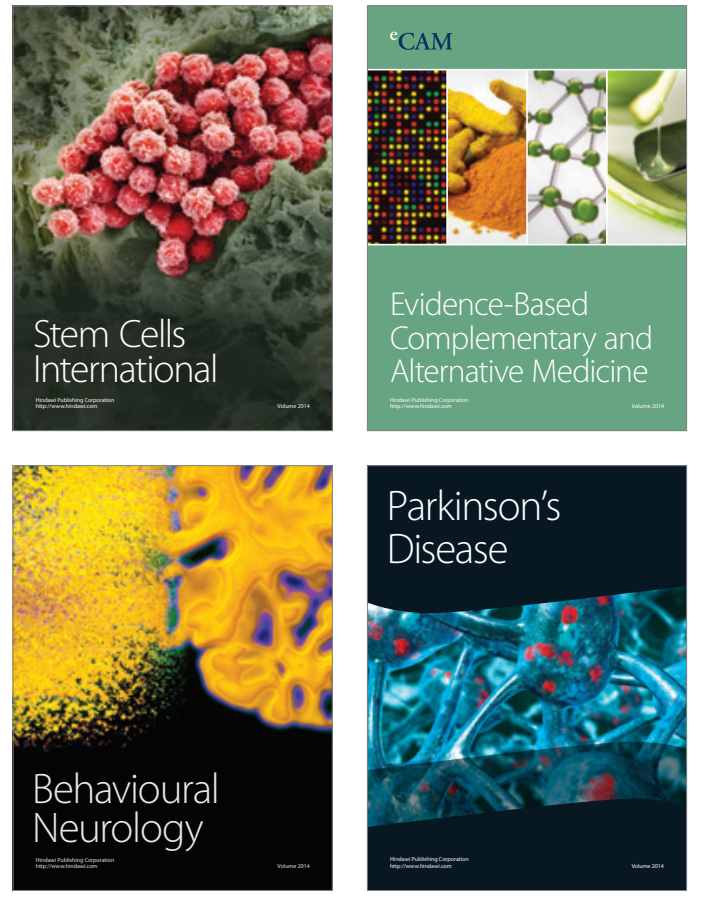
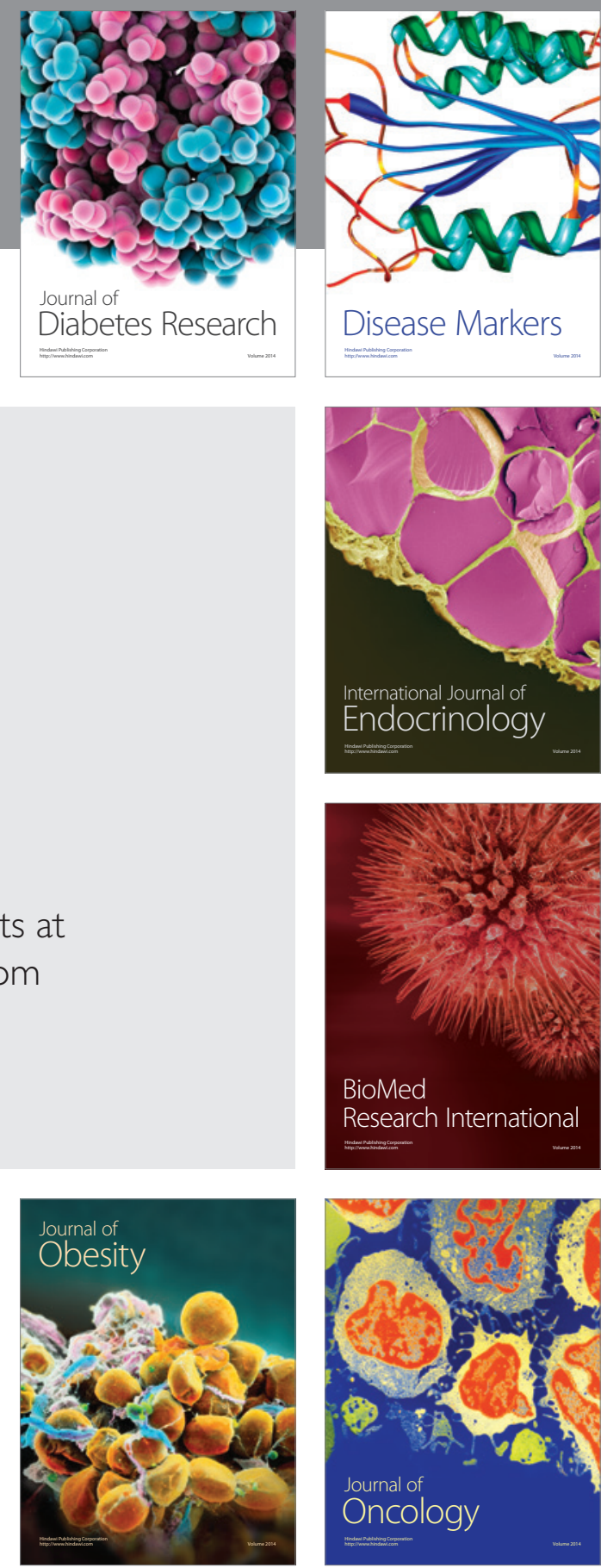

Disease Markers
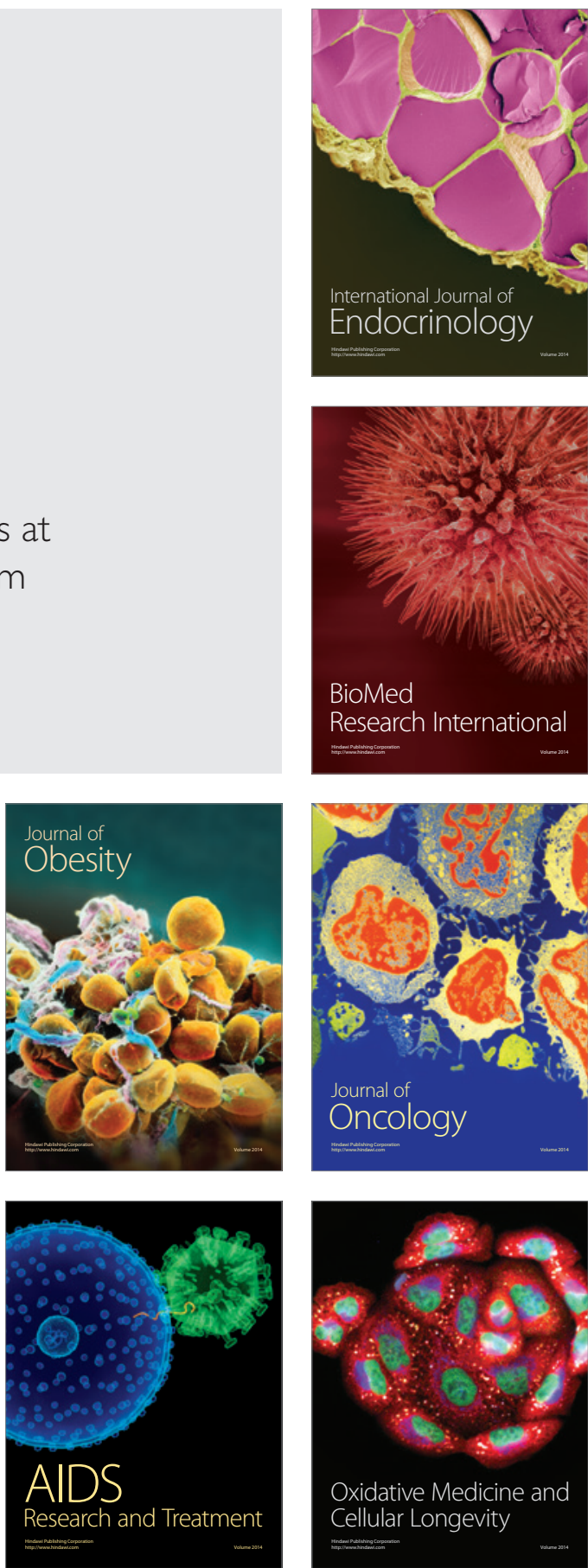